\title{
Operation Range of Generation Braking Force to Achieve High Efficiency Considering Combustion in a Free-Piston Engine Linear Generator System
}

\author{
Mitsuhide Sato, ${ }^{* * * a)}$ \\ Yuichiro Yamanaka* \\ Yinggang $\mathrm{Bu}^{*}$
}

Student Member,

Associate,

Member,

\author{
Masami Nirei ${ }^{* * *}$ \\ Hironobu Murata* \\ Tsutomu Mizuno*
}

\author{
Senior Member \\ Non-member \\ Senior Member
}

(Manuscript received Oct. 25, 2017, revised March 16, 2018)

\begin{abstract}
A linear generator system is expected to realize a highly efficient free-piston generation engine. The efficiency of the system mainly depends on the generation and thermal efficiencies of the engine. The generation efficiency decreases owing to a large braking force on the expansion stroke and a speed reduction at the ends of the stroke. This paper proposes generation control with resonance and output drop that improves the generation efficiency during the expansion stroke. When changing the amount of consumed fuel, the condition to maximize the electricity generation can be achieved by optimizing the ratio of the iron to copper losses. Furthermore, the proposed method improves the combustion performance of the engine by ensuring ideal speed at the combustion start position.
\end{abstract}

Keywords: current control, free piston engine, generation efficiency, linear generator, resonance

\section{Introduction}

In order to realize socially sustainable vehicles, improving the thermal efficiency of car engines is profoundly promoted by the Japanese Ministry of Internal Affairs and Communications. For this purpose, series hybrid systems (SHVs) are installed with an engine generator and a rechargeable battery. The rotary generators in this system are expected to realize high generation efficiency. In contrast, low maximum thermal efficiency and machine losses of crank mechanisms limit the system performances of conventional engines. The largest thermal and generation efficiencies of current SHVs are approximately $39 \%$ and $98 \%{ }^{(1)}$, respectively, and the estimated total system performance is approximately $38 \%$. To achieve high-efficiency hybrid vehicles, researchers have proposed a new car generator system called the free-piston engine linear generator (FPEG) system ${ }^{(2)-(6)}$.

The FPEG system is expected to incur fewer thermal losses and machine losses compared with SHVs. On the other hand, the generation efficiency of an FPEG system is lower than that of a rotary generator, which can continue generating electricity at a constant output. The efficiency of the FPEG system is reduced by the low speed at the end of the stroke

a) Correspondence to: Mitsuhide Sato. E-mail: mitsuhide@ele. pit-nagano.ac.jp

* Faculty of Engineering, Shinshu University

4-17-1, Wakasato, Nagano-city, Nagano 380-8553, Japan

** Nagano Prefectural Institute of Technology

813-8, Shimonogo, Ueda-city, Nagano 386-1211, Japan

*** National Institute of Technology, Nagano College

716, Tokuma, Nagano-city, Nagano 381-8550, Japan and the momentarily large braking force after combustion. In a previous study, the generation loss was reduced by a resonance and output drop control that optimizes the electricity generation by the expansion and compression trips ${ }^{(7)}$. Moreover, we reported on the design for linear generators by considering a large combustion force ${ }^{(8)}$. However, the setting method of the operating range of generation control with resonance and output drop was not investigated ${ }^{(7)}$. This paper clarifies the manner in which the setting operation ranges of generation control influence the resonance for reduction generation loss and improve top dead center (TDC) position precision.

\section{System Configuration and Conditions}

2.1 Structure Figure 1 shows the physical model of the FPEG system. The free piston is wrapped by a linear generator coil and installed with a permanent magnet to generate electricity. The free piston with the permanent magnet is pushed by combustion and spring repulsion forces. The coil generates electricity via induction, which charges the battery for the car driving. The piston movement is controlled by coordinating the braking force to the piston with an inverter.

The assumed equation of motion is given by Eq. (1). The TDC is defined at $x=0$ and the bottom dead center (BDC) $x_{\mathrm{b}}$ is free.

$$
m \frac{\mathrm{d}^{2} x}{\mathrm{~d} t^{2}}=F_{\mathrm{c}}-F_{\mathrm{g}}-F_{1}
$$

where $m$ and $x$ denote the mass and position of the piston, respectively, and $F_{\mathrm{c}}, F_{\mathrm{g}}$, and $F_{1}$ are the combustion, spring repulsion, and generation braking forces, respectively. 


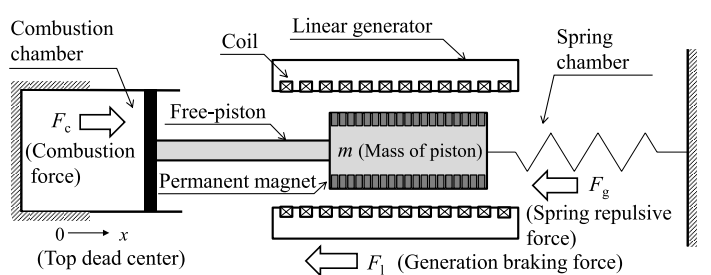

(a) Action model

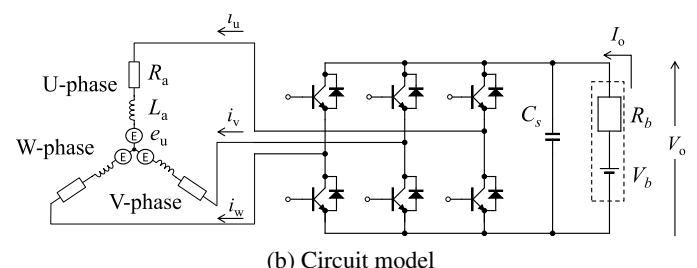

Fig. 1. Physical models of the FPEG system

2.2 Simulator The efficiency simulator of the FPEG system was constructed using the MATLAB-Simulink software. The linkages were defined by the motion and circuit equations. Figure 2 shows a block diagram of the FPEG simulator. The combustion force is calculated using Eq. (2). The combustion pressure $p_{\mathrm{c}}$ is given by Eq. (3) considering the sampling time in this simulation. The first term is based on Eq. (4) according to the governing equation of an adiabatic process. The second term indicates an additional pressure $\mathrm{d} p$ due to the combustion. The heat of combustion is converted into pressure using the first law of thermodynamics, as represented in Eq. (5). The heat release rate of combustion $\Delta P_{\mathrm{i}}$ continues for several milliseconds after passing the combustion start position $x_{\mathrm{s}}$. The combustion pressure is released at atmospheric pressure $\mathrm{p}_{\mathrm{a}}$ at the scavenging position $x_{\mathrm{a}}$ to simulate a two-stroke cycle engine, as represented in Eq. (3). The spring repulsion and generation braking forces are calculated by Eqs. (6) and (7), respectively. Figure 1(b) shows the equivalent circuit model of the FPEG system. The circuit model is solved for the current in the $\mathrm{U}, \mathrm{V}$, and $\mathrm{W}$ phases.

$$
\begin{aligned}
& F_{\mathrm{c}}(t)=A_{\mathrm{c}} \times p_{\mathrm{c}}(t) \\
& p_{\mathrm{c}}(t)= \begin{cases}p_{\mathrm{c}}(t-\Delta t) \times\left(\frac{V_{\mathrm{c}}(t-\Delta t)}{V_{\mathrm{c}}(t)}\right)^{\kappa}+\mathrm{d} p & \left(\text { if } x<x_{\mathrm{a}}\right) \\
\mathrm{p}_{\mathrm{a}} & \text { (if } \left.x>x_{\mathrm{a}}\right)\end{cases} \\
& \text { (N) } \cdots \cdots \cdots \cdots \cdots \cdots \\
& p=p_{0}\left(\frac{V_{0}}{p}\right)^{K} \\
& \mathrm{~d} p=\frac{\Delta P_{\mathrm{i}} \times\left(\Delta t / \Delta t_{\mathrm{Q}}\right) \times(\kappa-1)}{V_{\mathrm{c}}(t)} \\
& F_{\mathrm{g}}(t)=K_{\mathrm{g}} x+F_{\mathrm{a}} \\
& F_{1}(t)=K_{\mathrm{f}} i_{\mathrm{u}} \cos \left(\frac{\pi}{\tau} x\right)+K_{\mathrm{f}} i_{\mathrm{v}} \cos \left(\frac{\pi}{\tau} x-\frac{2 \pi}{3}\right) \\
& +K_{\mathrm{f}} i_{\mathrm{w}} \cos \left(\frac{\pi}{\tau} x-\frac{4 \pi}{3}\right)
\end{aligned}
$$

where $A_{\mathrm{c}}$ is the bore area, $V_{\mathrm{c}}$ is the volume of the combustion chamber, and $\kappa$ is the specific heat ratio. $\Delta t$ and $\Delta t_{\mathrm{Q}}$ are the sampling times of the simulator and the heat-release rate, respectively. $K_{\mathrm{g}}, F_{\mathrm{a}}$, and $K_{\mathrm{f}}$ denote the spring constant, spring thrust offset, and thrust constant, respectively. $i_{\mathrm{u}}, i_{v}$, and $i_{\mathrm{w}}$ are the currents of the $\mathrm{U}, \mathrm{V}$, and $\mathrm{W}$ phases, respectively, and $\tau$ is the pole pitch.

The generator current is controlled by a pulse-widthmodulated (PWM) signal with speed proportional-integral (PI) control, current PI control, vector control, and noninterference control. Figure 2 shows a block diagram for the current control. The PWM signal is generated by the triangle wave comparison method, and the carrier frequency is $10 \mathrm{kHz}$. In the vector control system, the generation braking force is proportional to the $q$-axis current, and Eq. (7) is replaced by Eq. (8). Here, the generation braking force is governed only by the electric current $i_{\mathrm{q}}$ along the $q$-axis in the vector space (under vector control, $i_{\mathrm{d}}=0$ ):

$$
F_{1}=\frac{3}{2} K_{\mathrm{f}} i_{\mathrm{q}}
$$

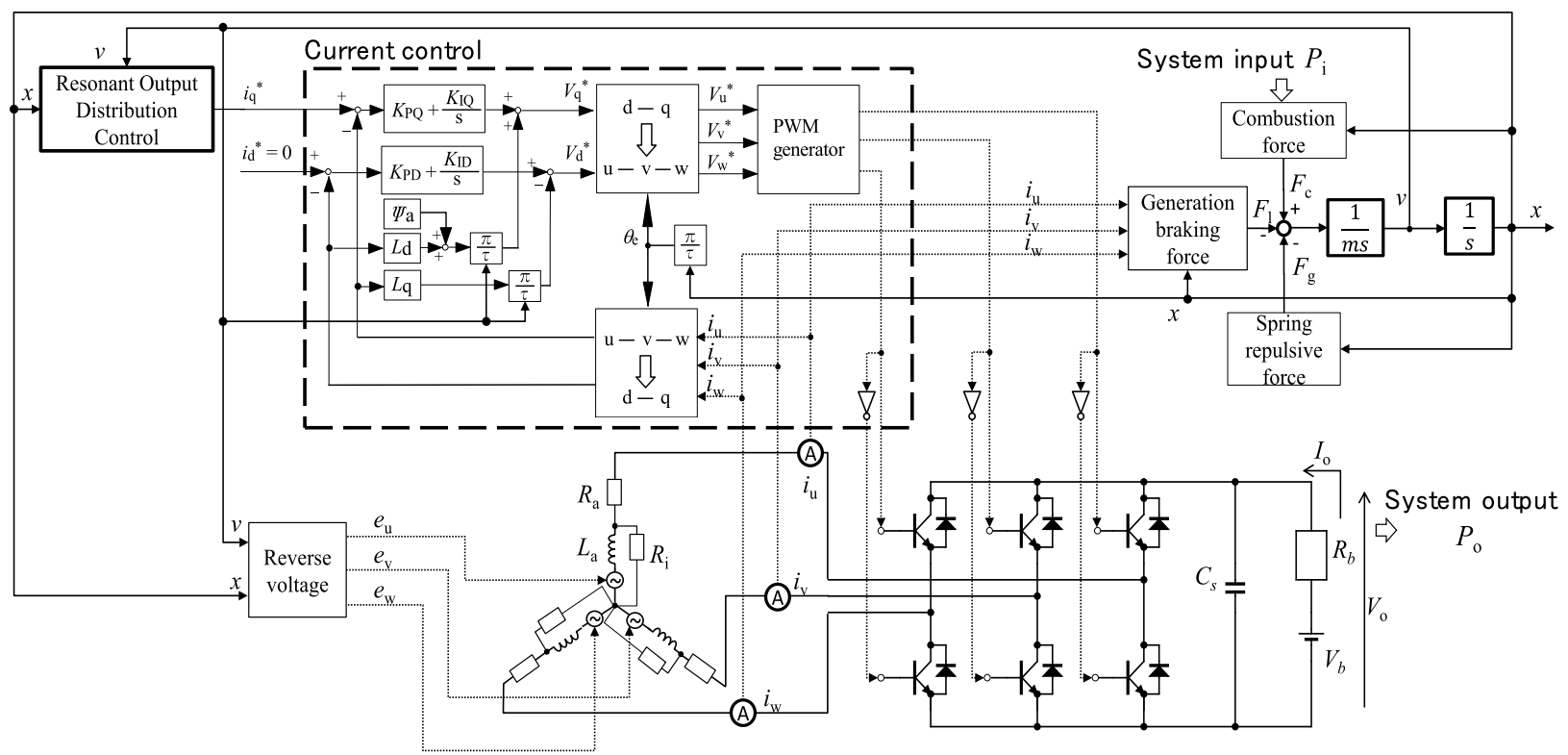

Fig. 2. Block diagram of the FPEG simulator 
where $i_{\mathrm{q}}$ is the $q$-axis current in the vector space.

The FPEG system was designed using the electromagnetic field analysis software JMAG-designer and SOLIDWORKS. The structure of the cylindrical linear generator is shown in Fig. 3. The permanent magnet adopts a Halbach structure. The generator constants, designed to reduce the copper loss and increase the thrust properties, are listed in Table $1^{(8)}$.

Figure 4 shows the energy balance in the proposed FPEG system. The fuel combustion heat $P_{\mathrm{i}}$ is the input and battery electricity $P_{\mathrm{o}}$ is the output. The losses are the exhaust thermal loss $Q_{\mathrm{t}}$, copper loss $Q_{\mathrm{c}}$, and iron loss $Q_{\mathrm{i}}$. The friction between a piston and a cylinder is primarily affected by the lubricating oil, which depends on the piston speed and the combustion chamber temperature ${ }^{(9)}$. However, assuming a friction value is difficult owing to various factors (such as cooling, and oil heat conduction). Therefore, this study does not consider the friction loss of the free piston $Q_{\mathrm{r}}$ while discussing improvements in the generation efficiency. The system efficiency $\eta_{\mathrm{s}}$ is calculated using Eq. (9), which considers the friction loss to be zero. The piston power $P_{\mathrm{p}}$ (Eq. (10)) is obtained by integrating the combustion force. The generation efficiency $\eta_{\mathrm{e}}$ is given by Eq. (11). The battery electricity is the output current multiplied by the output voltage. The switching loss of the inverter was assumed to be negligible for simplicity.

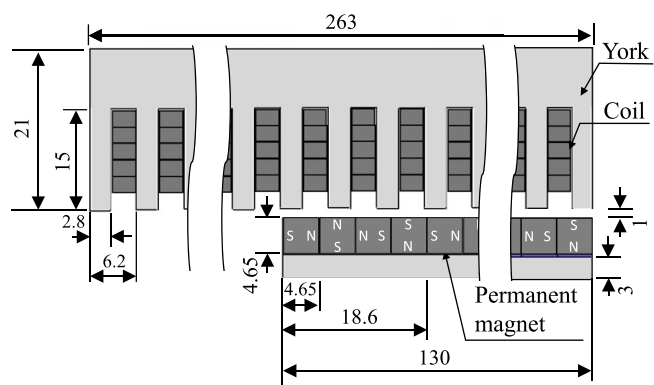

Fig. 3. Structure of the generator (unit: $\mathrm{mm}$ )

Table 1. Specifications of the generator

\begin{tabular}{l|c}
\hline \multicolumn{1}{c|}{ Item } & Value \\
\hline External dimensions & $\varnothing 168 \times 263(\mathrm{~mm})$ \\
\hline Dimensions of the mover & $\varnothing 128 \times 130(\mathrm{~mm})$ \\
\hline Mass of the mover & $5.25(\mathrm{~kg})$ \\
\hline Number of coil turns & 5 \\
\hline Resistance of the armature coil (per phase) & $108(\mathrm{~m} \Omega)$ \\
\hline Inductance of the armature coil (per phase) & $1.8(\mathrm{mH})$ \\
\hline Thrust constant & $22(\mathrm{~N} / \mathrm{A})$ \\
\hline
\end{tabular}

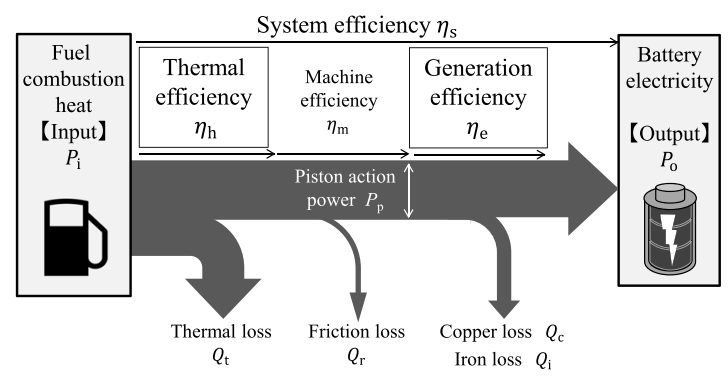

Fig. 4. Energy flow in the FPEG system

$$
\begin{aligned}
\eta_{\mathrm{s}} & =\frac{P_{\mathrm{o}}}{P_{\mathrm{i}}} \times 100=\frac{P_{\mathrm{i}}-Q_{\mathrm{t}}-Q_{\mathrm{r}}-Q_{\mathrm{c}}-Q_{\mathrm{i}}}{P_{\mathrm{i}}} \times 100 \\
& \approx \frac{P_{\mathrm{i}}-Q_{\mathrm{t}}}{P_{\mathrm{i}}} \times \frac{P_{\mathrm{i}}-Q_{\mathrm{t}}-Q_{\mathrm{c}}-Q_{\mathrm{i}}}{P_{\mathrm{i}}-Q_{\mathrm{t}}} \times 100 \\
& =\frac{\eta_{\mathrm{h}}}{100} \times \frac{\eta_{\mathrm{e}}}{100} \times 100 \quad(\%) \ldots \ldots \ldots \ldots \ldots \ldots \ldots \ldots \ldots \\
P_{\mathrm{p}} & =f \times \int F_{\mathrm{c}} \mathrm{d} x \quad(\mathrm{~W}) \cdots \ldots \ldots \ldots \ldots \\
\eta_{\mathrm{e}} & =\frac{P_{\mathrm{o}}}{P_{\mathrm{p}}} \times 100=\frac{I_{\mathrm{o}} \times V_{\mathrm{o}}}{P_{\mathrm{p}}} \times 100 \quad(\%) \cdots \cdots \cdots
\end{aligned}
$$

where $\eta_{\mathrm{h}}$ is the thermal efficiency, and $f$ is the piston frequency.

\section{Resonant Output Distribution Control}

3.1 Principal Immediately after combustion, the copper loss in the FPEG system increases as a result of speed reduction at the end of the stroke and the large braking force at the time of the expansion trip. When the generation braking force is proportional to the piston speed, the operating range as seen on the efficiency map is described by line-(1) in Fig. 5. The generation efficiency is approximately $90 \%$, accounting for the copper loss alone ${ }^{(7)}$. The generation efficiency is thought to be reduced by electricity generation in the low-efficiency domain. Figure 5 also shows a characteristic curve (the force-velocity curve) that is based on the capacity of the generator and inverter. The $d$-axis current for field weakening is necessary to prevent the piston from being uncontrollably beyond the characteristic curve ${ }^{(10)}$.

In this study, the resonant output distribution (ROD) control is defined, line-(2), as shown in Fig. 5. This control characteristic is resonant, and the maximum output drop nearly transfers the output from an expansion to a compression trip using the piston inertia and spring force, as shown in Fig. $6^{(7)}$. An ROD is expected for a similar operation of the rotary generator, which can maintain almost constant output and high efficiency. Furthermore, this control does not generate electricity at low velocity at the motion edge on the efficiency drop area owing to the reciprocating movement peculiar to a linear motion system. Therefore, the ROD control effectively reduces the copper loss. The total generation efficiency also increases according to the operating generation in the high efficiency area line-(2), as shown in Fig. 5. The operating

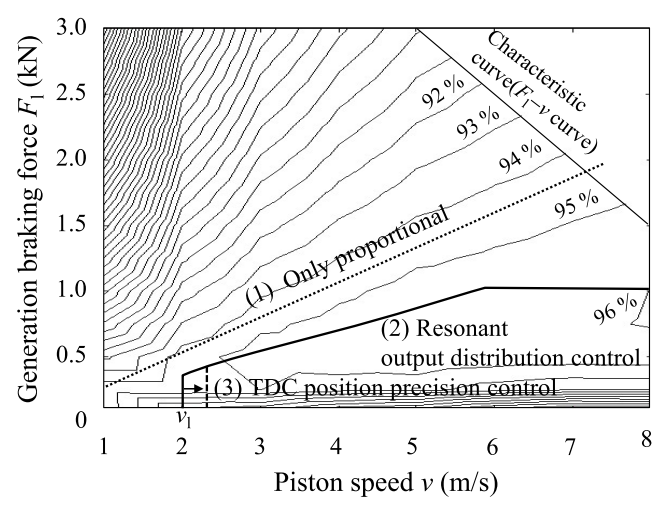

Fig. 5. Operating range of the FPEG system with ROD control on the efficiency map 


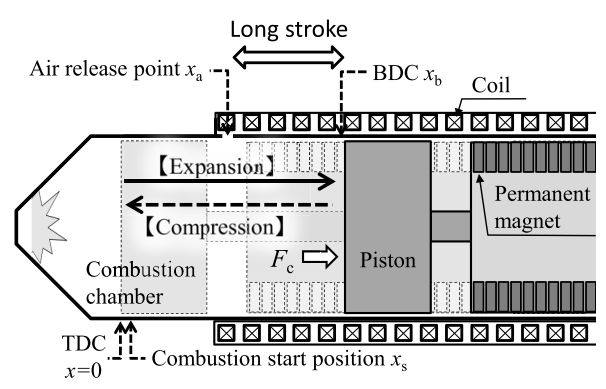

Fig. 6. Effect of a long stroke with ROD control

range is given by Eq. (12), and the operation line of the ROD control is decided via the following methods ${ }^{(7)}$.

1) The generation braking force $K_{1}$ is set considering the approach to a resonance state by the spring-massdamping model, which is very similar to this system.

2) The maximum generation braking force $F_{1-\max }$ is set to generate a high efficiency area and an operation line not beyond the $F_{1}-v$ curve in the efficiency map. (The method to decide $F_{1-\text { max }}$ by considering the $F_{1}-v$ curve is described in Section 4.1.)

3) The generation velocity limit $v_{1}$ is set considering the piston reciprocating continuously and the efficiency drop in the low velocity at the motion edge in the efficiency map. (The method to decide $v_{1}$ taking into account the TDC position accuracy is described in Section 4.2.)

The ROD control can distribute the generated output from the expansion stroke to the compression stroke via extension of the stroke using the resonance. The power generation of the expansion stroke and the compression stroke are equalized by suppressing the maximum generation braking force. Furthermore, the $d$-axis current for the field weakening control is unnecessary by setting the operation line not beyond the $F_{1}-v$ curve in the efficiency map. For these reasons, the generation efficiency can be reduced.

$$
F_{1}= \begin{cases}0 & \left(v<v_{1}\right) \\ K_{1} v & \left(v>v_{1}\right) \\ F_{1-\text { max }} & \left(F_{1}>F_{1-\max }\right)\end{cases}
$$

where $K_{1}$ is the generation braking constant, $v$ is the piston velocity, and $F_{1-\max }$ is the maximum generation braking force.

3.2 Effect Figure 7 and Table 2 show the simulation results of the FPEG with the ROD control. The heat release ratio, is given at combustion start position at every 1 stroke, which means system input, as shown in Fig. 7(a). As the piston reciprocates, the combustion force follows an Otto cycle (Figs. 7(b)-(d)). Decreasing the spring constant increases the stroke movement by $30 \mathrm{~mm}$. The ROD effect clearly appears in Fig. 7(e), which means the high generation braking force on the expansion stroke is limited. The difference of maximum output power between each stroke was lower with the ROD than without the ROD as in Fig. 7(f), and the compression-stroke generation was higher than without the ROD. The copper loss was reduced despite the high combustion output. Accounting for both copper and iron losses, the generation efficiency $\eta_{\mathrm{e}}$ increases up to $95 \%$.

The $d$-axis current is kept near zero as a secondary effect, i.e., not beyond the characteristic curve with the ROD. In this system, a large generation braking force is necessary at the time of high-speed piston movement without the ROD because an instantaneous and large combustion energy is input into a linear generator. Therefore, the operation range must

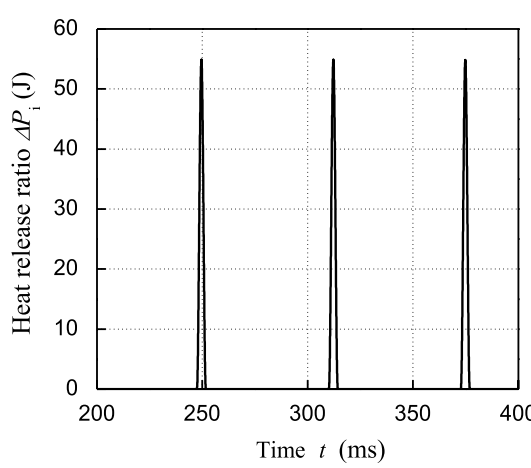

(a) Heat release ratio

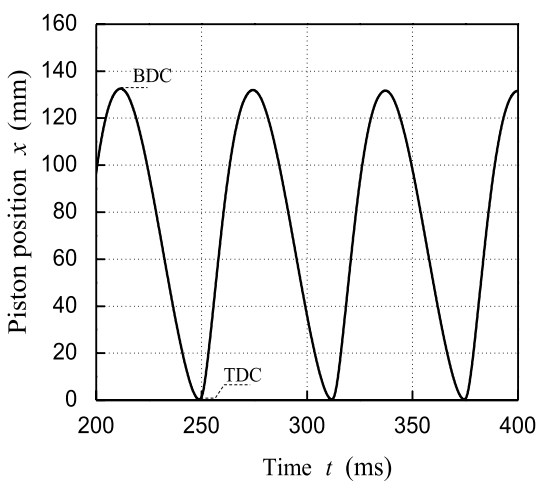

(d) Piston position

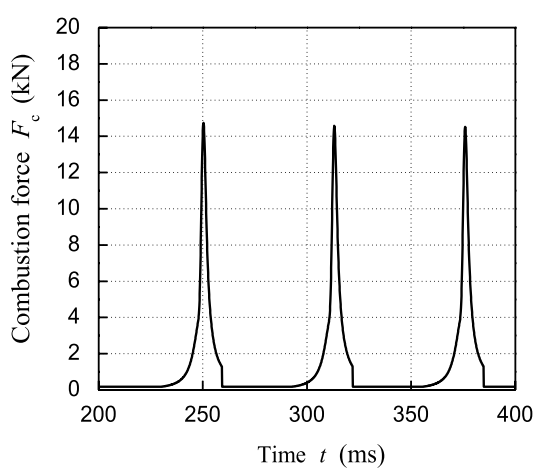

(b) Combustion force (Time dependence)

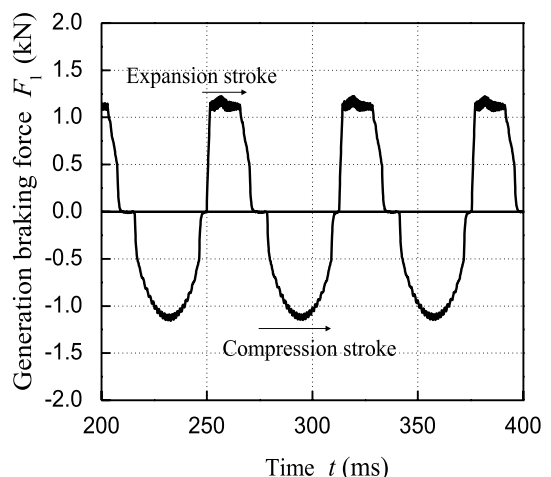

(e) Generation braking force

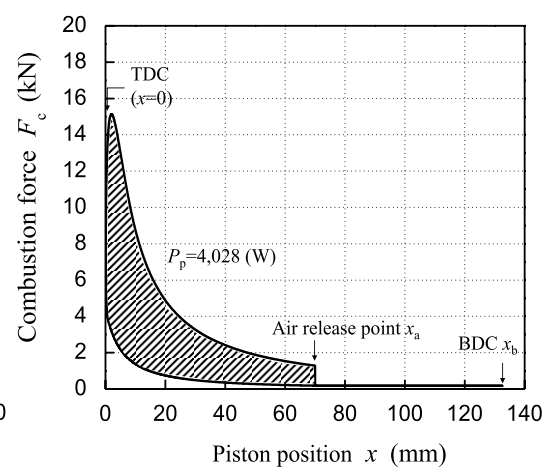

(c) Combustion force (Position dependence)

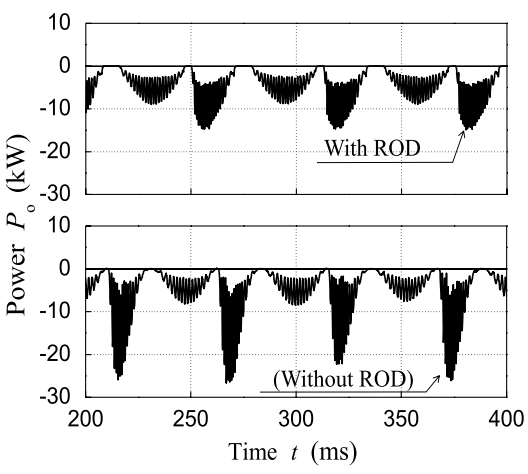

(f) Power of output

Fig. 7. Simulation results with the ROD control 
Operation Range to Achieve High Efficiency in FPEG (Mitsuhide Sato et al.)

Table 2. Simulation results with the ROD control

\begin{tabular}{l|c|c}
\hline \multicolumn{1}{c|}{ Item } & Symbol & Value \\
\hline \hline Fuel combustion heat & $P_{\mathrm{i}}$ & $8,558(\mathrm{~W})$ \\
\hline Piston action power & $P_{\mathrm{p}}$ & $4,028(\mathrm{~W})$ \\
\hline Battery electricity & $P_{\mathrm{o}}$ & $3,840(\mathrm{~W})$ \\
\hline Thermal efficiency & $\eta_{\mathrm{h}}$ & $47.07(\%)$ \\
\hline Generation efficiency & $\eta_{\mathrm{e}}$ & $95.32(\%)$ \\
\hline System efficiency & $\eta_{\mathrm{s}}$ & $44.87(\%)$ \\
\hline
\end{tabular}

not be considered beyond the $F_{1}-v$ curve. The larger battery voltage and generator size can transfer the $F_{1}-v$ curve to a higher output area. However, an upper limit level exists while considering car deployment. Therefore, the ROD can optimize the operation area of the generated braking force within the $F_{1}-v$ curve to maintain higher generation efficiency. The effect of this control is remarkable in the case of low friction. A setting considering not only generation loss but also friction loss is required in an actual machine.

3.3 Estimation of Generation Efficiency In practice, the amount of fuel consumption for electricity generation is affected by factors, such as system capacity. Therefore, the generation efficiency trends were investigated while reducing the fuel amount.

The piston motion approximately follows Eq. (13), indicating that the FPEG system is similar to a forced-dampedvibration model in the dynamics. This system is nearly in the resonant state because combustion is constantly triggered near the TDC during the compression stroke. Therefore, the piston position, stroke extent, and average piston speed are described by Eqs. (14), (15), and (16), respectively.

The copper loss reduction when restraining the burning energy is explained as follows by this model. When the combustion energy reduces by one-half, the combustion force and the generation braking constant must also be halved by Eq. (15), and the generation braking force is restrained by maintaining the piston stroke uniformity. The $q$-axis current is controlled to one-half and copper loss is one-quarter by Eqs. (8) and (17), respectively. The copper loss is inversely proportional to the square of the input energy by maintaining the same stroke.

We now explain why the iron loss is unaffected by the burning energy. The iron loss is caused by the magnetic flux with the magnets and the armature current. Figure 8 shows the influence of iron loss by flux linkage's in a generation braking current changing at constant speed in the finite element method (FEM) analysis. The iron loss by the magnetic flux of the permanent magnets is dominant because the effect by the armature current is small, as shown in Fig. 8. The magnetic flux with the electric current nearly crosslinks at the edge of the armature teeth and cancels the magnetic flux with the magnets ${ }^{(8)}$. Therefore, the iron loss is nearly zero at the edge of the low current area. Conversely, in most of the armature except the edges, iron loss occurs almost entirely via the magnetic flux with the magnets. The influence of the permanent magnets is dominant in the entire generator.

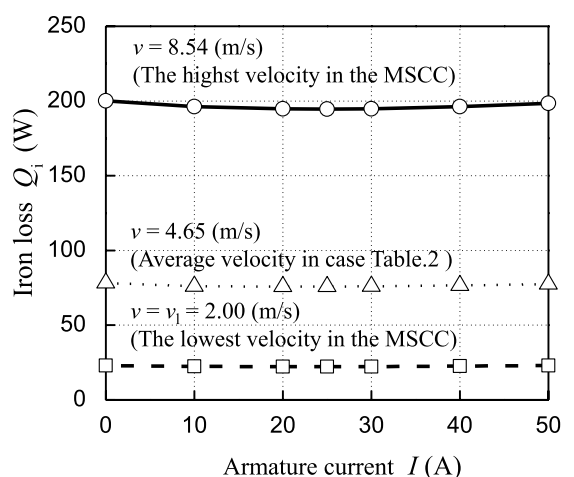

Fig. 8. Dependence of the iron loss on the armature current

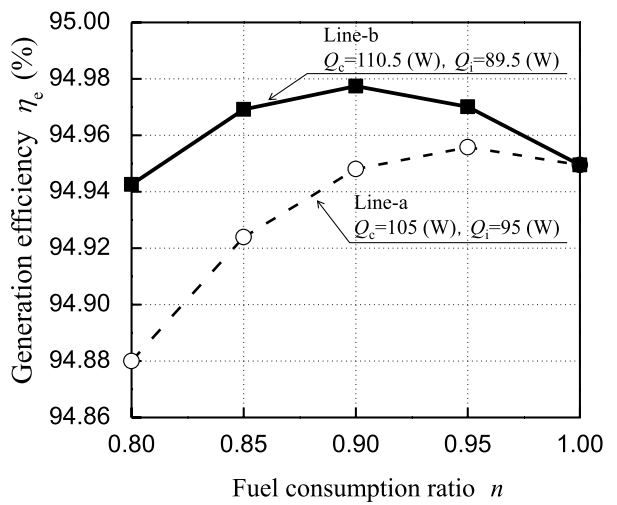

Fig. 9. Efficiency versus the fuel consumption ratio

The iron loss via the magnetic flux of the permanent magnets is mainly proportional to the velocity. Therefore, the iron loss is nearly unchanged when restraining the burning energy because the average velocity remains constant when maintaining the same stroke.

Finally, the generation efficiency is discussed. The generation efficiency accounts for the copper and iron losses. It can also be expressed in terms of the fuel consumption ratio $n$ (Eq. (18)). In general, the piston work $P_{\mathrm{p}}$ is almost proportional to the amount of fuel consumed. The maximum generation efficiency is accomplished when minimizing the copper and iron losses with Eq. (19). Eq. (20) is the condition of maximum generation efficiency. Figure 9 plots the generation efficiency versus $n$ calculated by Eq. (20) under the conditions of lines-a and b on Fig. 9. For the standard amount of fuel $(n=1)$, the copper and iron losses sum to $200 \mathrm{~W}$ under both conditions. Under the conditions of line (b), the inflection point of the efficiency appears at $n=0.9$. When changing the amount of consumed fuel, the condition to maximize the electricity generation exists by optimization the ratio of the iron to copper losses.

$$
\begin{aligned}
& m \ddot{x}+K_{1}{ }^{\prime} \dot{x}+K_{\mathrm{g}}{ }^{\prime} x=f(t)=F_{0} \sin \omega t \quad(\mathrm{~N}) \cdots \cdots \\
& x=x_{\mathrm{b}}{ }^{\prime} \sin (\omega t+\delta) \quad(\mathrm{m}) \\
& x_{\mathrm{b}}{ }^{\prime}=\frac{1}{K_{\mathrm{l}}{ }^{\prime}} \sqrt{\frac{m}{K_{\mathrm{g}}}} F_{0} \\
& \text { (m) } \\
& v_{\mathrm{ave}}=4 f x_{\mathrm{b}}=\frac{2 F_{0}}{\pi K_{\mathrm{l}}{ }^{\prime}} \\
& Q_{\mathrm{c}}=i_{\mathrm{q}}^{2} R_{\mathrm{a}} \\
& \text { (W) }
\end{aligned}
$$




$$
\begin{gathered}
\eta_{\mathrm{e}}(n)=\frac{n P_{\mathrm{p}}-n^{2} Q_{\mathrm{c}}-Q_{\mathrm{i}}}{n P_{\mathrm{p}}} \times 100 \\
=\frac{P_{\mathrm{p}}-n Q_{\mathrm{c}}-\frac{1}{n} Q_{\mathrm{i}}}{P_{\mathrm{p}}} \times 100 \\
\frac{\mathrm{d}}{\mathrm{d} n}\left(n Q_{\mathrm{c}}+\frac{1}{n} Q_{\mathrm{i}}\right)=0 \ldots \ldots \ldots \ldots \ldots \ldots \ldots \\
Q_{\mathrm{i}}=n^{2} Q_{\mathrm{c}} \quad(\mathrm{W}) \ldots \ldots \ldots \ldots \ldots \ldots
\end{gathered}
$$

where $K_{\mathrm{l}}{ }^{\prime}(\mathrm{N} / \mathrm{m} / \mathrm{s})$ and $K_{\mathrm{g}}{ }^{\prime}(\mathrm{N} / \mathrm{m})$ are the damping and spring constants, respectively. $x_{\mathrm{b}}$ ' is the piston stroke $(\mathrm{m}), \omega$ ' is the angular frequency of the external force $(\mathrm{rad} / \mathrm{s}), \delta$ is the phase contrast $(\mathrm{rad} / \mathrm{s}), v_{\text {ave }}$ ' is the average piston velocity $(\mathrm{m} / \mathrm{s})$, and $f$ ' is the frequency of the moving piston $(\mathrm{Hz})$ in a forceddamped-vibration model. $R_{\mathrm{a}}$ is the armature resistance $(\Omega)$.

\section{Method for Setting the Operating Range Ac- counting for Higher System Performance}

4.1 Consideration of Characteristic Curve The operation line of generation braking force should be restrained within the characteristic curve because increasing electric current along the $d$-axis for weakening field (line (1) on Fig. 5, which is beyond the characteristic curve). Therefore, a setting of operation line should consider the characteristic curve.

Considering the energy balance in the system shown in Fig. 4, the piston reciprocates as defined sine curve in Eq. (14). In particular, the piston work $W_{\mathrm{p}}$ must be absorbed by the work of generation braking $W_{1}$ during one stroke for maintaining the reciprocation, as shown in Eq. (21). The piston velocity (Eq. (22)) is obtained by differentiating the vibration-damping model (Eq. (14)). Using Eqs. (22) and (12) with ROD, the generation braking work in Eq. (21) can be re-expressed as Eq. (23). Furthermore, assuming non sine curve movement of the piston, the velocity of Eq. (22) can be solved by differentiating the piston position $x(t)$. Eq. (23) is satisfied when the piston moves inside the characteristic curve under the conditions of Fig. 7 with ROD. This equation is effective when setting of operation line.

$$
\begin{aligned}
& W_{\mathrm{p}}=W_{1}=\int F_{1} \mathrm{~d} x \\
& v=\frac{\mathrm{d} x}{\mathrm{~d} t}=\omega x_{\mathrm{b}} \cos (\omega t+\delta) \\
& =\omega x_{\mathrm{b}} \frac{\sqrt{x_{\mathrm{b}}^{2}-x^{2}}}{x_{\mathrm{b}}}=\omega \sqrt{x_{\mathrm{b}}^{2}-x^{2}} \quad(\mathrm{~m} / \mathrm{s}) \cdots \cdots \\
& W_{\mathrm{l}}=\int_{-x_{\mathrm{b}} / 2}^{x_{\mathrm{b}} / 2}\left(K_{\mathrm{l}} \omega \sqrt{x_{\mathrm{b}}^{2}-x^{2}}+F_{1-\max }\right) \mathrm{d} x \\
& =\left[\left(\frac{K_{1} \omega}{2} x \sqrt{x_{\mathrm{b}}^{2}-x^{2}}+x_{\mathrm{b}}{ }^{2} \sin ^{-1} \frac{x}{x_{\mathrm{b}}}+F_{1-\max } x\right)\right]_{-x_{\mathrm{b}} / 2}^{x_{\mathrm{b}} / 2} \\
& \approx \frac{\sqrt{3} K_{1} \omega}{4} x_{\mathrm{b}}{ }^{2}+F_{1-\max } x_{\mathrm{b}}
\end{aligned}
$$

4.2 Consideration of TDC The TDC position affects the combustion performance and the heat loss in the ideal Otto cycle as described in Eq. $(24)^{(11)}$. As the thermal efficiency is large, the TDC position is small. However, if the TDC becomes very small, the combustion performance degrades because the volume of the minimum combustion

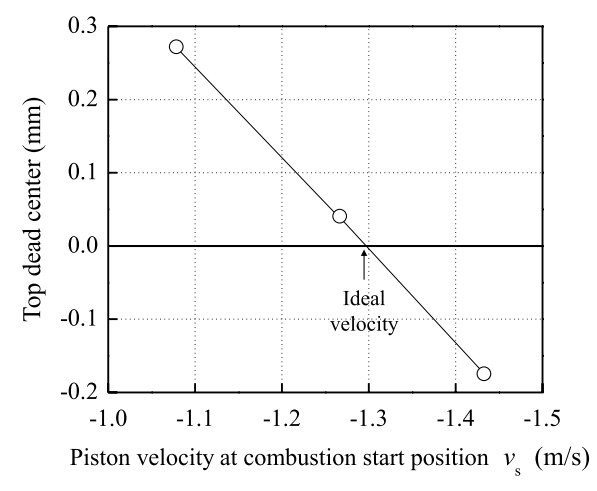

Fig. 10. Relationship between piston velocity at the combustion start position and TDC.

chamber decreases considerably; the temperature inside the chamber increases significantly. Therefore, the ideal TDC position for high thermal efficiency is $x \approx 0$. When the TDC position shifts by $1 \mathrm{~mm}$ from the standard position, the thermal efficiency improves or drops by $0.2 \%$, following Eq. (24). However, millimeter-order control is difficult to achieve. In addition, low-speed generation control restrains the generation at the stroke edge, thereby reducing the generation efficiency. Therefore, the setting method is required to improve accuracy in the TDC position determination and precision of repetition.

$$
\eta_{\mathrm{t}}{ }^{*}=1-\left(\frac{V_{\mathrm{t}}}{V_{\mathrm{a}}}\right)^{\kappa_{\mathrm{c}}-1} \times 100 \quad(\%) \cdots \ldots \ldots \ldots \ldots
$$

where $\eta_{\mathrm{t}}{ }^{*}$ is the theoretical thermal efficiency, $V_{\mathrm{t}}$ and $V_{\mathrm{a}}$ are the volumes of the combustion chamber at the TDC and the air release point, respectively, and $\kappa_{\mathrm{c}}$ is the specific heat ratio during combustion.

Herein, the inertia at the combustion start position is adjusted toward the ideal state. Figure 10 shows the relationship between the piston speed at the combustion start position $v_{\mathrm{s}}$ and the TDC position. If the piston approaches the TDC at high speed, it can easily be under the zero point. To maintain the TDC position near zero, the speed at the combustion start position needs to be optimized. In the ideal Otto cycle, the combustion process is isothermal heating, but in actual operation, the thermogenesis rate changes over time. This simulation model includes a time-dependent thermogenesis rate, which is similar to real combustion. An increase in inertia is expected to change the piston behavior and the TDC position, enlarging the piston speed at the combustion start position. The piston movement can be adjusted toward the most suitable speed at the combustion start position by changing the speed domain of the compression stroke based on the BDC.

The TDC position precision control is suitable for this purpose, as shown on line (3) in Fig. 5, which sets the variable generation velocity limit $v_{1}$ depending on a BDC position $x_{\mathrm{b}}$. On the compression stroke, the piston work derives from the elastic energy saved in the spring chamber on the expansion stroke. Eq. (25) is the energy conservation law between the BDC position $x_{\mathrm{b}}$ and the combustion start position $x_{\mathrm{s}}$ on the compression stroke. The energy conservation law should be used to set the generation velocity limit $v_{1}$. The elastic energy $W_{\mathrm{g}}$ at $\mathrm{BDC}$ can be written as Eq. (26). The generation 
braking work $W_{1}$ can be written as Eq. (27) because the generation braking force is proportional to only the piston speed and the maximum velocity is low during the compression stroke. The advantage of this control is dependent on only time at the generation velocity limit $t\left(v_{1}\right)$. The work acting on the combustion chamber during the compression stroke $W_{\mathrm{c}}$ is given by Eq. (28) with the adiabatic compression model ${ }^{(11)}$. This term is constant because the air release position and the combustion start position are fixed. $W_{\mathrm{s}}$ is the kinetic energy of the piston at the combustion start position in Eq. (29). $v_{\mathrm{s}}$ is the ideal speed at the combustion start position, as shown in Fig. 10. Therefore, the generation area can be set by Eq. (25) with only deciding variable $v_{1}$ depending on a BDC position.

As a result of simulation, this control can obtain the almost ideal piston action; a velocity of $1.28 \mathrm{~m} / \mathrm{s}$ at the combustion start position and a TDC position of $0.02 \mathrm{~mm}$. The piston action successfully improved the thermal efficiency by setting the speed domain. In addition, setting the generation domain based on the BDC and speed information could provide a generalizable index for optimizing engine performance.

$$
\begin{aligned}
& W_{\mathrm{g}}-W_{1}-W_{\mathrm{c}}=W_{\mathrm{s}} \\
& \text { (J) } \cdots \cdots \cdots \cdots \cdots \cdots \cdots \cdots \cdots(25) \\
& W_{\mathrm{g}}=\int F_{\mathrm{g}} \mathrm{d} x=\frac{1}{2} K_{\mathrm{g}} x_{\mathrm{b}}^{2}+\int_{x_{\mathrm{s}}}^{x_{\mathrm{b}}} F_{\mathrm{a}} \mathrm{d} x \\
& W_{1}=\int F_{1} \mathrm{~d} x=\int_{t\left(v_{1 \_\mathrm{b}}\right)}^{t\left(v_{1-\mathrm{t}}\right)} K_{1} v \times v \mathrm{~d} t=\int_{t\left(v_{1 \_}\right)}^{t\left(v_{1-\mathrm{b}}\right)} K_{1} v^{2} \mathrm{~d} t \\
& \text {................ } \\
& W_{\mathrm{c}}=\int F_{\mathrm{c}} \mathrm{d} x=F_{\mathrm{c}}\left(x_{\mathrm{s}}\right) x_{\mathrm{s}}{ }^{\kappa} \int_{x_{\mathrm{s}}}^{x_{\mathrm{a}}} x^{\kappa} \mathrm{d} x=\text { const } \\
& W_{\mathrm{s}}=\frac{1}{2} m v_{\mathrm{s}}^{2} \\
& \text { (J) }
\end{aligned}
$$

where $t\left(v_{\mathrm{l}_{-}}\right)$and $t\left(v_{\mathrm{l}_{-} \mathrm{b}}\right)$ are the time passing at the generation velocity limit TDC-side and BDC-side on compression stroke, respectively.

\section{Conclusion}

This paper proposed a method for improving the electricity generation efficiency of an FPEG system. The ROD control reduces the generation losses, which transfers the quantity of generation output from the expansion stroke to compression stroke using a resonant and maximum output drop. The control is highly effective in systems with large work input such as combustion forces. By largely reducing the generation losses, the generation efficiency increased up to $95 \%$ with the ROD control. The tendencies of the copper and iron losses under the ROD were clarified, and a best point yielding the largest efficiency under the ROD was determined by changing the fuel consumption amount.

Furthermore, to improve the system performance, the action area must remain within the characteristic curve of the linear generator, and the TDC position must approach zero as closely as possible. The method in this study easily determines the combustion energy and the conditions under which the ROD will operate within the characteristic curve. The method sets the generation domain of the compression trip based on the BDC. Consequently, the speed at the combustion start position is close to ideal, and the error in the TDC is very small.

\section{Acknowledgment}

This work was partly supported by TAKEUCHI MFG. CO., LTD. research grant.

\section{References}

( 1 ) R. Mizutani, T. Tachibana, M. Masayuki, K. Akatsu, and N. Hoshi: "Electric Drive Technologies Contribution to Low-Fuel-Comsumption Vehicles", IEEJ Trans. Ind., Vol.135, No.9, pp.894-891 (2015)

( 2 ) H. Kosaka, T. Akita, K. Moriya, S. Goto, Y. Hotta, T. Umeno, and K. Nakakita: "Development of Free Piston Engine Linear Generator System (First Report) Investigation of Fundamental Characteristics", Trans. Soc. Automative Engineers of Japan, Vol.45, No.4, pp.683-689 (2014)

( 3 ) K. Moriya, S. Goto, H. Kosaka, T. Akita, Y. Hotta, T. Umeno, and K. Nakakita: "Development of Free Piston Engine Linear Generator System (Second Report) Investigation of Control System for Generator", Trans. Soc. Automative Engineers of Japan, Vol.45, No.4, pp.691-697 (2014)

( 4 ) C. Yuan, H. Feng, Y. He, and J. Xu: "Combustion Characteristics Analysis of a Free-Piston Engine Generator Coupling with Dynamics and Scavenging", Energy, Vol.102, pp.637-649 (2016)

( 5 ) H. Yan, D. Wang, and Z. Xu: "Design and Simulation of Opposed-Piston Four-Stroke Free-Piston Linear Generator", SAE Technical Paper, 2015-011277 (2015)

( 6 ) J. Wang, M. West, D. Howe, H.Z.L. Parra, and W.M. Arshad: "Design and Experimental Verification of a Linear Permanent Magnet Generator for a Free-Piston Energy Converter", IEEE Trans. Energy. Convers., Vol.22, No.2, pp.299-306 (2007)

( 7 ) M. Sato, M. Nirei, Y. Yamanaka, H. Murata, Y. Bu, and T. Mizuno: "Examination of a Free-Piston Engine Linear Generator System with Generation Control for High Efficiency", Proceedings of the 11th LDIA2017 (International Symposium on Linear Drives for Industry Applications), CT-2 (2017)

( 8 ) Y. Yamanaka, M. Nirei, M. Sato, H. Murata, Y. Bu, and T. Mizuno: "Design of Linear Synchronous Generator Suitable for Free-Piston Linear Generator System", Proceedings of the 11th LDIA2017 (International Symposium on Linear Drives for Industry Applications), TD-2 (2017)

( 9 ) Y. Wakuri, M. Soejima, T. Kitahara, M. Nunotani, and M. Ootsubo: "Studies on the Characteristics of Piston Ring Friction", Trans. of the Jap. Soc. of Mech. Eng. C, Vol.58, No.551, pp.2293-2298 (1992)

(10) Y. Komatsu, M. Naka, H. Yamada, T. Kobayashi, and T. Okada: "Maximum Output Operation by Equivalently Weakening Field and Optimal Parameters of Brushless DC Motor", T. IEE Japan, Vol.113-D, No.3, pp.349-356 (1993)

(11) Jpn. Soc. Mech. Eng.: "JSME Textbook Series Thermodynamics", From Jpn. Soc. Mech. Eng. (2002)

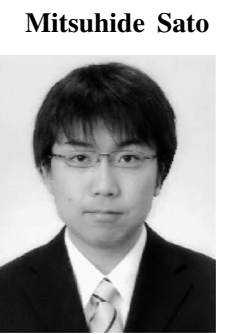

Student Member) received his B.E. and M.E. degrees from the Tohoku University, Sendai, Japan, in 2009 and 2011, respectively. From 2011 to 2015, he worked as an electrical engineer at the TOSHIBA Corp., in Japan. Since 2015, he has been working as a Lecturer with the Nagano Prefectural Institute of Technology, Ueda, Japan. Now he is currently a Ph.D. student at the Shinshu University, Nagano, Japan. His research interests include linear synchronous generator and power electronics. He is a student member of the Japan Society of Applied Electromagnetics and Mechanics (JSAEM)

Masami Nirei (Senior Member) is currently a professor in the Depart-

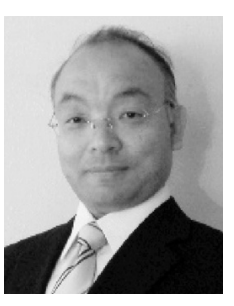
ment of Electronics and Computer Science at the National Institute of Technology, Nagano College. He received his B.E., M.E., and D.E. degrees in electrical engineering from the Shinshu University. He worked as an engineer in the Motor section of the Gunma NEC from 1988 to 1990 . He joined the Shinshu University Hospital in 1990 as an assistant professor and was transferred to the National Institute of Technology, Nagano College, in 1991. His research work includes electromagnetic actuators and sensors, optimal design, and numerical analysis. He is a member of the IEEE, the Magnetics Society of Japan, and the Japan Society of Applied Electromagnetics and Mechanics. 
Yuichiro Yamanaka (Associate) received his B.E. degree from the

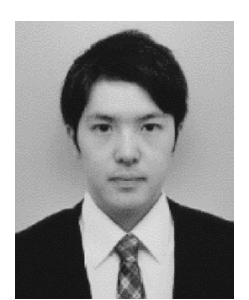
Shinshu University, Nagano, Japan, in 2017. He is currently an M.E. student at the Shinshu University, NagaNo. His research interests include linear synchronous generators.
Hironobu Murata (Non-member) received his B.E. degree from

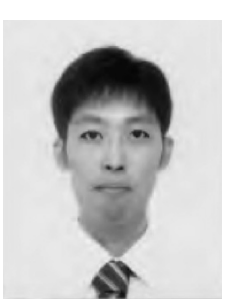

Yinggang Bu

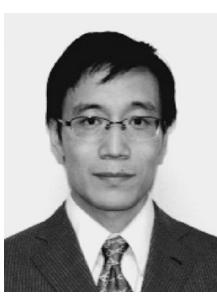

Member) received his B.S. degree in Electrical Engineering from the Taiyuan University of Technology, China, in 2002. He received his M.S. and Ph.D. degrees in Electrical and Electronic Engineering from the Shinshu University, Japan, in 2006 and 2009, respectively. He is currently an associate professor in the Department of Engineering at the Shinshu University, Japan. His research interests include linear motors and actuators, as well as wireless power transfer. He is a member of the IEEE and the Institute of

Electrical Engineers of Japan.

Tsutomu Mizuno (Senior Member) received his B.E. and M.E. de-

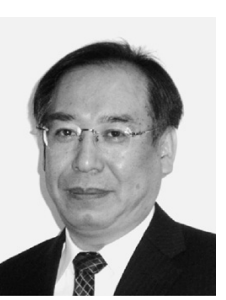
grees from the Shinshu University, Nagano, Japan, in 1981 and 1983, respectively. From 1983 to 1996, he worked as an electrical engineer at the AMADA CO., LTD. in Japan. From 1996 to 1999, he worked as an assistant professor; from 1999 to 2011, he worked as an associate professor. Since 2011, he has been working as a professor in the Department of Electrical and Computer Engineering, Shinshu University, Nagano, Japan. He holds a Doctor of Engineering degree from the Shinshu University. His research interests include linear motors, linear actuators, and electromagnetic sensors. He is a member of the IEEE, the Magnetics Society of Japan, and the Japan Society of Applied Electromagnetics and Mechanics (JSAEM). 